\title{
MONTE CARLO ANALYSIS OF THE BACKSCATTERING OF RADIATION FROM A SPHERE TO A PLANE
}

\author{
W. R. MARTIN \\ Department of Nuclear Engineering, University of Michigan, Ann Arbor, MI 48109-2104, U.S.A. \\ G. C. Pomraning \\ School of Engineering and Applied Science, University of California at Los Angeles, \\ Los Angeles, CA 90024-1597, U.S.A.
}

(Received 21 July 1989)

\begin{abstract}
A Monte Carlo method is described for computing the backscattering of radiation from a sphere situated above an emitting plane. Numerical results are given and compared with analytic bounds for this problem which were previously reported in this journal.
\end{abstract}

\section{INTRODUCTION}

In an earlier paper in this journal,' a class of problems was considered involving radiative transfer in a halfspace consisting of emitting, absorbing and scattering matter, and occupying $-\infty<z \leqslant 0$. The adjoining halfspace was vacuum, except for a sphere located some distance above the matter surface at $z=0$. The question was posed as to whether the influence of the sphere is sufficiently strong to substantially affect the radiative transfer in the matter. In that paper, an idealized version of this class of problems was studied to partially quantify the answer to this question. Specifically, the problem treated was the infinite $x-y$ (or $r-\phi$ ) plane located at $z=0$, emitting a time-independent axisymmetric flux, $F_{\mathrm{E}}(r)$, into a vacuum. The emitted radiation was assumed to be isotropic (obey Lambert's law). A purely scattering sphere of radius $R$ was located above the plane, with the center of the sphere at $x=y=0$ and $z=h$, with $h \geqslant R$. The scattering interaction was assumed to be isotropic and characterized by a scattering cross section $\sigma$ which was taken to be uniform throughout the sphere. The quantity of interest, as a function of the radial coordinate $r$, was $F_{\mathrm{R}}(r)$, the radiative flux reflected by the sphere back to the plane at $z=0$.

In the earlier paper,' we defined the reflectance ratio $\Gamma(r)$ by the expression

$$
\Gamma(r)=F_{\mathrm{R}}(r) / F_{\mathrm{E}}(r)
$$

and decomposed $\Gamma(r)$ into a collisional factor $1-\exp (-\tau)$ and a geometric factor $G(r)$, according to

$$
\Gamma(r)=[1-\exp (-\tau)] G(r) .
$$

Here $\exp (-\tau)$ is defined as the fraction of the photons impinging upon the sphere which pass through the sphere without making a collision. It was argued that to a reasonable approximation $\tau$ could be computed using the simple Dirac expression for the mean chord length, ${ }^{2}$ i.e.,

$$
\tau \simeq \tau_{\mathrm{D}}=4 \sigma R / 3 .
$$

The geometric factor $G(r)$ arises from the details of transporting the photons from the plane through the vacuum to the sphere via viewfactors, accounting for the multiple scattering collisions in the sphere, and then transporting the photons back to the plane, again via viewfactors. The complexity of this problem, in particular the details of the transport in the sphere, precludes an analytic solution for $G(r)$ in any generality. In the earlier paper, $G(r)$ was bounded at $r=0$ by analytic considerations corresponding to two limiting cases of an optically thin sphere $(\tau \ll 1)$ and an optically thick sphere $(\tau \gg 1)$. These two bounds for $G(0)$ are roughly a factor of two apart. 
In the present paper we revisit this problem and develop a Monte Carlo method for computing exactly, aside from statistical errors, the reflectance ratio, $\Gamma(r)$. Decomposing our results according to Eq. (2), we can then compare essentially exact results for $G(0)$ with the previously reported bounds. Our Monte Carlo results also give an indication of the accuracy of using Eq. (3) to estimate the noncollision probability, $\exp (-\tau)$. Section 2 of this paper gives the details of the Monte Carlo method, including several biasing schemes employed to reduce the statistical error. Representative numerical results are given in Sec. 3. A short Appendix is included which develops an interpolation model to estimate $G(0)$ from the analytic bounds for optically thin and thick spheres.

\section{MONTE CARLO METHODOLOGY}

In principle, simple analog Monte Carlo could be used in an entirely straightforward manner to solve the problem of interest here. However, such a technique would in general involve a prohibitive amount of computational time even on the most powerful computers. Accordingly, we have introduced several variance reduction techniques which we discuss here. Also discussed in this section are the sampling and particle tracking techniques we have used, as well as a tallying strategy involving a point detector estimator for computing the reflectance ratio, $\Gamma(r)$, at the point $r=0$ (directly under the sphere). As in the earlier paper, ${ }^{1}$ we take $\Gamma(0)$ to be the primary quantity of interest since $\Gamma(r)$ should be in general be a maximum at $r=0$.

The first item we discuss is source biasing. In general, it is desirable to emit photons from the plane surface in the direction of the sphere to maximize the number of important trajectories. In doing this, however, the photon weight must be adjusted accordingly. If we define $w_{0}$ to be the analog weight and assume photons are only sampled from the solid angle element $\Delta \boldsymbol{\Omega}$, the adjusted weight $w$ of the sampled photon is given by

$$
w=w_{0} P(\Delta \Omega),
$$

where $P(\Delta \Omega)$ is the probability that the $\Delta \Omega$ phase space volume would have been selected in the analog simulation. In the present application, photons are emitted uniformly, i.e., according to Lambert's law, into the upper hemisphere $(z>0)$. To implement this, we consider the sphere of radius $R$ located a height $h$ above the plane $z=0$, and define a coordinate system such that the coordinates of the center of the sphere are $x=0, y=y_{0}$, and $z=h$. Here the position $y_{0}$ is sampled, and thus every photon is tracked in a local coordinate system with its origin at the point of emission. This choice of the coordinate system, shown in Fig. 1, simplifies the subsequent algebraic considerations.

In this coordinate system, it is easily shown that the sphere subtends a polar angle range $\left[\theta_{2}, \theta_{1}\right]$, where

$$
\theta_{2}=\theta-\Delta \theta / 2, \quad \theta_{1}=\theta+\Delta \theta / 2
$$

with $\Delta \theta$ given by

$$
\Delta \theta=2 \cos ^{-1}\left[L /\left(y_{0}^{2}+h^{2}\right)^{1 / 2}\right]
$$

where $L$ is the tangential distance to the sphere according to

$$
L^{2}=y_{0}^{2}+h^{2}-R^{2} \text {. }
$$

The corresponding range for $\mu=\cos \theta$ subtended by the sphere is $\mu_{1} \leqslant \mu \leqslant \mu_{2}$, with

$$
\mu_{1}=\left(h L-y_{0} R\right) /\left(y_{0}^{2}+h^{2}\right), \quad \mu_{2}=\mu_{1}+\Delta \mu,
$$

where

$$
\Delta \mu=2 y_{0} R /\left(y_{0}^{2}+h^{2}\right)
$$




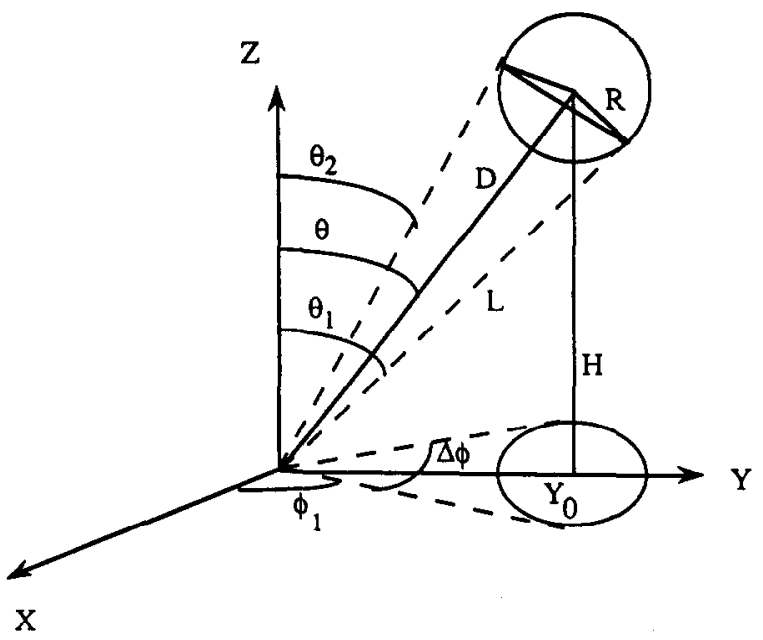

Fig. 1. The geometry of the sphere over the plane.

If $y_{0} \leqslant R, \mu_{2}$ in Eq. (8) is replaced by $\mu_{2}=1$. To find the range in azimuthal angle, $\phi_{1} \leqslant \phi \leqslant \phi_{2}$, subtended by the sphere, we note that the sphere is centered over the $y$ axis. This gives

$$
\phi_{1}=(\pi / 2)-(\Delta \phi) / 2 ; \quad \phi_{2}=(\pi / 2)+(\Delta \phi) / 2,
$$

where

$$
\Delta \phi=2 \sin ^{-1}\left(R / y_{0}\right) .
$$

To sample $\mu$ and $\phi$, the probability density distribution functions must be normalized according to the above limits on the variables. Since the emitted intensity is isotropic, $p(\mu)$ is linear in $\mu$ and $p(\phi)$ is uniform in $\phi$ according to, applying the normalizations,

$$
p(\mu)=2 \mu /\left(\mu_{2}^{2}-\mu_{1}^{2}\right) ; \quad p(\phi)=\left(\phi_{2}-\phi_{1}\right)^{1} .
$$

Using these density functions, the corresponding cumulative distribution functions are formed and then inverted to obtain the sampled $\mu$ and $\phi$ as a function of the random number $\xi$ drawn uniformly on $0 \leqslant \xi \leqslant 1$. This gives

$$
\mu=\left[\mu_{1}^{2}+\left(\mu_{2}^{2}-\mu_{1}^{2}\right) \xi / 2\right]^{1 / 2} ; \quad \phi=\phi_{1}+\xi \Delta \phi .
$$

The corresponding weight $w$ of the sampled photon emitted into $(\mu, \phi)$ is given by Eq. (4), where $p(\Delta \Omega)$ is the probability that the photon would have been emitted from the solid angle element $\Delta \boldsymbol{\Omega}$. This is clearly given by

$$
p(\Delta \mathbf{\Omega})=\left(\mu_{2}^{2}-\mu_{1}^{2}\right)\left(\phi_{2}-\phi_{1}\right) /(2 \pi) .
$$

The use of this source-biasing technique, i.e., sampling $\mu$ and $\phi$ from Eq. (13), ensures that all photons emitted from the $z=0$ plane will hit the sphere.

This source biasing technique works very well in the present application, saving a substantial amount of computational time over a simple analog Monte Carlo for most cases. As we shall see in the next section, this saving is roughly between a factor of 1 and 10 . However, it will tend to favor particles sampled far from the origin in some cases, since all photons emitted, no matter what the point of origin, will hit the sphere. This is evident by noting that the emitted intensity of radiation in an annulus of width $\mathrm{d} r$ located at $r$ is given by $(2 \pi r \mathrm{~d} r) F_{\mathrm{E}}(r)$. Thus if $F_{\mathrm{E}}(r)$ falls off with distance slower than $1 / r$, source biasing forces most collisions with the sphere to occur with photons originating at large $r$. Although the source biasing method is unbiased in that the mean is preserved by adjusting the weight, this preferential treatment of large $r$ may increase the statistical error in the result. To adjust for this potential problem, Russian roulette was considered as a way of rejecting some particles emitted far away from the sphere. This rejection was done, somewhat arbitrarily, with a probability proportional to the solid angle subtended by the sphere at that 
distance. Specificially, we introduce the ratio of the subtended solid angle at $r$ to the subtended solid angle at $r=2 R$, i.e.,

$$
\operatorname{ratio}(r)=P_{\mathrm{r}}(\Delta \Omega) / P_{2 \mathrm{R}}(\Delta \Omega) \text {. }
$$

We reject any particle emitted at $r$ if a random number $\xi>\operatorname{ratio}(r)$. If the particle is not rejected, its weight is multiplied by $1 /$ ratio $(r)$. For $r \leqslant 2 R$, no Russian roulette is used. This simple strategy introduces a computational saving of, roughly speaking, another factor between 1 and 10 as we shall see in the next section.

The next item we discuss is sampling (in physical space) of the photons emitted by the plane. In the earlier paper, ${ }^{1}$ three spatial distributions were considered for the emitted radiation, namely

$$
\begin{gathered}
F_{\mathrm{E}}^{(1)}(r)=C_{1}, \\
F_{\mathrm{E}}^{(2)}(r)=C_{2} h /\left(h^{2}+r^{2}\right)^{1 / 2}, \\
F_{\mathrm{E}}^{(3)}(r)=C_{3} R /\left(R^{2}+r^{2}\right)^{1 / 2},
\end{gathered}
$$

where the $C_{i}$ are constants. We note that $p_{i}(r) \equiv r F_{\mathrm{E}}^{(i)}(r)$ is just the probability density function for emittance of a source photon at radial coordinate $r$. (The factor $r$ in this product accounts for the factor $r$ in the area factor $2 \pi r \mathrm{~d} r$.) if we assume, as the earlier paper did, that Eqs. (16)-(18) hold in the interval $0 \leqslant r \leqslant K h$ with $K$ a specified constant, and take $F_{\mathrm{E}}^{(i)}(r)$ identically zero for $r>K h$, we can impose the required condition that the integral of $p_{i}(r)$ over all $r$ is unity by a proper choice of the constants $C_{i}$. We find

$$
\begin{gathered}
1 / C_{1}=(K h)^{2} / 2, \\
1 / C_{2}=h^{2}\left[\left(1+K^{2}\right)^{1 / 2}-1\right], \\
1 / C_{3}=R^{2}\left[\left(1+K^{2} h^{2} / R^{2}\right)^{1 / 2}-1\right] .
\end{gathered}
$$

For the numerical calculations summarized in the next section, we chose $K=10$ as in the previously reported results.' As noted earlier, we use these $p_{i}(r)$ to sample the position of the sphere rather than the emitted particle.

Forming the cumultative distribution functions corresponding to the $p_{i}(r)$ and inverting these expressions gives, in the usual way, the sampled radial coordinates as a function of $\xi$, a random number uniformly distributed in the interval $0 \leqslant \xi \leqslant 1$. In our thrce cases we find

$$
\begin{gathered}
r_{1}=\left(2 \xi / C_{1}\right)^{1 / 2}, \\
r_{2}=\left[\left(2 \xi / C_{2}\right)+\xi^{2} /\left(C_{2} h\right)^{2}\right]^{1 / 2}, \\
r_{3}=\left[\left(2 \xi / C_{3}\right)+\xi^{2} /\left(C_{3} R\right)^{2}\right]^{1 / 2} .
\end{gathered}
$$

This distance $r_{i}$ is taken as $y_{0}$, the $y$ coordinate of the center of the sphere as seen by a photon at the origin.

The difficulty associated with particle tracking in Monte Carlo simulations is determining the intersection of a straight line particle trajectory with interfaces between materials. In our case this is particularly simple since the only interface of concern is that between the sphere and the vacuum. Given a starting point of a trajectory either on the surface of or within the sphere, it is a simple matter to find the intersection of this trajectory with the surface of the sphere. One need only find the roots of a simple quadratic. The distance to collision, $x$, is given in terms of a random number $\xi$ by the standard formula

$$
x=-\sigma^{-1} \ln (1-\xi) .
$$

If the point of collision corresponding to $x$ is within the sphere, the photon is scattered and starts a new trajectory in a random direction corresponding to an isotropic scattering phase function. If the distance $x$ is beyond the surface of the sphere, the photon has left the sphere and travels unimpeded in the vacuum either back to the surface $z=0$ or into deep space. Only those photons which strike the $z=0$ surface are of interest since only these contribute to $F_{\mathrm{R}}(r)$.

It is clear that for optically thin spheres $(\tau \ll 1)$ only a very few photons incident upon the sphere will suffer a collision within the sphere. This causes very large statistical fluctuations, which can 
be alleviated by using a forced collision technique for the first flight path. This variance reduction strategy is based upon the observation that the probability for a collision within a distance $x$ can be written as the product of two probabilities according to

$$
P(x)=P(x \mid D) P(D) .
$$

Here $P(D)$ is the unconditional probability of a collision within a distance $D$, and $P(x \mid D)$ is the conditional probability for a collision within a distance $x$, given that a collision has occurred within $0 \leqslant x \leqslant D$. Noting that $P(x \mid D)$ is a cumulative distribution function and that $P(D)=1-\exp (-\sigma D)$, we find in the usual way that the sampled distance to collision $x$ is given in terms of a random number $\xi$ according to

$$
x=-\sigma^{-1} \ln \{1-\xi[1-\exp (-\sigma D)]\} .
$$

This is implemented in an unbiased fashion by adjusting the weight accordingly. To do this, the source photon is split into two photons, one of which collides inside the sphere, and one which passes through the sphere without a collision. These two photons have weights determined by the probabilities of these events, namely

$$
w_{\text {unc }}=w_{0} \exp (-\sigma D) ; \quad w_{\mathrm{c}}=w_{0}[1-\exp (-\sigma D)],
$$

where $D$ is the distance to the sphere boundary and $w_{0}$ is the photon weight prior to the splitting. These two separate photons would normally be followed, each with its own weight. Since we apply this forced collision technique only to the first flight path, the uncollided photon can never contribute to the reflected flux $F_{\mathrm{R}}(r)$; hence we need not follow this photon. This forced collision technique can give a computational saving of up to a factor of 100 , as we shall see in the next section.

Since we are interested in the reflected flux directly under the sphere $(r=0)$, we consider a small circular area in the $z=0$ plane centered at $r=0$ (the tally surface), and compute $W$, the total weight of all photons which cross this surface. This gives an estimate of the reflected flux (per unit area) at $r=0$ in the $z=0$ plane as

$$
F_{\mathrm{R}}(0)=W /(N A),
$$

where $A$ is the area of the tally surface and $N$ is the total number of photons emitted from the $z=0$ source plane. We note that for resolution purposes, one would want to take $A$ to be very small. However, if this were done not many photons would cross the tally surface, resulting in large statistical errors. Thus there are conflicting requirements in the definition of the tally area. For our numerical calculations summarized in the next section, we chose $A=\pi R^{2} / 100$.

The problem of conflicting requirements in the size of the tally surface can be alleviated by employing a point detector estimator. This scheme is based upon the observation that the product of the speed of light $c$ and the photon density $\rho$ at point $\mathbf{r}$ due to a unit isotropic point source at $\mathbf{r}^{\prime}$ is given by the well known point source kernel

$$
c \rho_{\mathrm{pl}}(\mathbf{r})=\left(4 \pi s^{2}\right)^{-1} \exp [-\alpha(s)],
$$

where $s=\left|\mathbf{r}-\mathbf{r}^{\prime}\right|$ and $\alpha(s)$ is the number of photon mean free paths between the source point $\mathbf{r}^{\prime}$ and the detector point $\mathbf{r}$. The Monte Carlo simulation is creating the collision source within the sphere, and each collision can be viewed as an isotropic point source with the potential of contributing to the density (and flux) at point $r$. Noting that a given photon may constitute more than one point source due to multiple collisions, we define $F_{n}(\mathbf{r})$ as the total (over all collisions) contribution to the flux at point $\mathbf{r}$ due to photon $n$. We have, taking the point of interest to be $\mathbf{r}=0(r=z=0)$,

$$
F_{n}(0)=c w_{n} \sum_{i} \rho_{\mathrm{pt}, i} \cos \theta_{i},
$$

where $w_{n}$ is the weight of the $n$th photon colliding in the sphere, and the summation over $i$ is a summation over collisions. The factor $c \cos \theta_{i}$, where $\theta_{i}$ is the angle between the vector $\mathbf{r}^{\prime}$ and a normal to the plane at $z=0$, accounts for the fact that our quantity of interest is the one way flux, 
$F_{\mathrm{R}}(0)$, rather than the photon density $\rho$. Finally, we need sum $F_{n}(0)$ over all contributing photons to obtain the reflected flux, i.e.,

$$
F_{\mathrm{R}}(0)=\sum_{n} F_{n}(0)
$$

This point detector estimator is idcally suited to our application since we are interested in the reflected flux, $F_{\mathrm{R}}(r)$, at a specific position, namely $r=0$. In our numerical calculations, the use of this point estimator rather than a tally surface achieved computational savings of a factor as large as 2500 .

An indication of the statistical error in our Monte Carlo simulations can be achieved by applying the usual formula for the standard deviation to $F_{\mathrm{R}}(0)$. Denoting this standard deviation by $\Delta F_{\mathrm{R}}(0)$, we have

$$
\left[\Delta F_{\mathrm{R}}(0)\right]^{2}=\frac{1}{N(N-1)}\left\{\sum_{n=1}^{N} F_{n}^{2}(0)-\frac{1}{N}\left[\sum_{n=1}^{N} F_{n}(0)\right]^{2}\right\}
$$

where $N$ is the number of histories (source particles emitted by the $z=0$ plane).

Thus our Monte Carlo methodology has the capability of employing four different variance reduction techniques, namely source biasing, Russian roulette, forced first collision, and a point detector estimator. Using all of these variance reduction techniques, $N$ in Eq. (32), the number of histories, is of the order of $10^{4}$ to achieve a $1 \% \mathrm{SD}$, although this number varied substantially depending upon the parameters $\sigma, R$, and $h$ of the problem under consideration.

\section{NUMERICAL RESULTS}

The reflected flux directly under the sphere, $r=0$, was computed using the Monte Carlo techniques described in the last section. The reflectance ratio, $\Gamma(0)$, was then formed according to Eq. (1) and subsequently decomposed into a first collision probability, $1-\exp (-\tau)$, and a geometric factor, $G(0)$, according to Eq. (2). To make this decomposition, we considered three choices for the optical depth $\tau$. These are the simple Dirac optical chord length value given by Eq. (3); an average value of $\tau$ defined as

$$
\tau_{\mathrm{av}}=\sigma l_{\mathrm{av}},
$$

where $l_{a v}$ is an average geometric chord length, computed as the weighted (photon weight) average over the first flights for all photons which impinge upon the sphere; and an effective value of $\tau$ defined as

$$
\tau_{\text {eff }}=-\ln \langle\exp (-\tau)\rangle,
$$

where $\langle\exp (-\tau)\rangle$, the average exponential, is the weighted average again taken over all first flights of photons which strike the sphere. The use of $\tau_{\text {eff }}$ for $\tau$ in Eq. (2) gives the actual value for the first flight collision probability. Both $\tau_{\mathrm{av}}$ and $\tau_{\mathrm{eff}}$ were computed as part of the Monte Carlo simulations, and can be compared to the simple Dirac formula given by Eq. (3) as we shall do later on in this section. Once the emittance function, $F_{E}(r)$, is specified, there are three parameters which define a particular problem, namely $\sigma, R$, and $h$. Thus there are two independent dimensionless parameters, and we take these to be $\sigma R$ and $h / R$.

To test the accuracy of the optically thin bound for $G(0)$ proposed earlier by analytic considerations, ${ }^{1}$ we performed Monte Carlo calculations for a very thin sphere, $\sigma R=0.01$, as a function of $h / R$. These calculations used all four of the variance reduction techniques discussed in the last section to compute $F_{\mathrm{R}}(0)$, and hence $\Gamma(0)$ according to Eq. (1). To obtain $G(0)$, we used Eq. (3) with $\tau$ given by $\tau_{\text {eff }}$ as defined by Eq. (34). The resulting $G(0)$ then accounts for the first flight collision probability in an exact (except for statistical errors) way. The results for the geometric factor directly under the sphere, $G(0)$, are shown in Table 1 . We see that for a constant emittance function as given by Eq. (16), the Monte Carlo results agree, to within $1 \mathrm{SD}$, with the analytic thin sphere bound for all values of $h / R$. This agreement demonstrates, more than anything else, that the Monte Carlo calculations are being performed correctly since one can easily argue that for this constant emittance function the analytic result must be exact as $\sigma R$ becomes vanishingly small. This argument is based upon the observation that the photon density in the 
Table 1. Comparison of the thin sphere analytic bound with Monte Carlo simulations for $\sigma R=0.01$

\begin{tabular}{|c|c|c|c|}
\hline $\begin{array}{c}\text { Emittance } \\
\text { Function } \\
\mathbf{F}_{\mathbf{E}}(\mathbf{r})\end{array}$ & $\mathbf{h} / \mathbf{R}$ & $\begin{array}{c}\text { Analytic } \\
\text { Bound for } \\
\text { G(0) }\end{array}$ & $\begin{array}{c}\text { Monte Carlo } \\
\text { Result for } \\
\text { G(0) }\end{array}$ \\
\hline Eq. (16) & $\begin{array}{r}2 \\
4 \\
6 \\
8 \\
10\end{array}$ & $\begin{array}{l}0.113 \\
0.0281 \\
0.0125 \\
0.00704 \\
0.00450\end{array}$ & $\begin{array}{c}0.114 \pm 0.001 \\
0.0281 \pm 0.0001 \\
0.0125 \pm 0.0001 \\
0.00700 \pm 0.00004 \\
0.00449 \pm 0.00003\end{array}$ \\
\hline Eq. (17) & $\begin{array}{r}2 \\
4 \\
6 \\
8 \\
10\end{array}$ & $\begin{array}{l}0.0619 \\
0.0155 \\
0.00688 \\
0.00387 \\
0.00248\end{array}$ & $\begin{array}{c}0.0648 \pm 0.0005 \\
0.0157 \pm 0.0001 \\
0.00692 \pm 0.00003 \\
0.00387 \pm 0.00002 \\
0.00246 \pm 0.00001\end{array}$ \\
\hline Eq. (18) & $\begin{array}{r}2 \\
4 \\
6 \\
8 \\
10\end{array}$ & $\begin{array}{l}0.0414 \\
0.00621 \\
0.00197 \\
0.000863 \\
0.000452\end{array}$ & $\begin{array}{c}0.0445 \pm 0.0003 \\
0.00636 \pm 0.00003 \\
0.00198 \pm 0.00001 \\
0.000862 \pm 0.000005 \\
0.000451 \pm 0.000003\end{array}$ \\
\hline
\end{tabular}

Table 2. Comparison of the thick sphere analytic bound with Monte Carlo simulations for $\sigma R=50$

\begin{tabular}{|c|r|l|l|}
\hline $\begin{array}{c}\text { Emittance } \\
\text { Function } \\
\mathbf{F}_{\mathbf{E}}(\mathbf{r})\end{array}$ & h/R & $\begin{array}{c}\text { Analytic } \\
\text { Bound for } \\
\mathbf{G ( 0 )}\end{array}$ & $\begin{array}{c}\text { Monte Carlo } \\
\text { Result for } \\
\mathbf{G}(\mathbf{0})\end{array}$ \\
\hline Eq. (16) & 2 & 0.231 & $\begin{array}{c}0.225 \pm 0.003 \\
\end{array}$ \\
& 4 & 0.0537 & $\begin{array}{l}0.0521 \pm 0.0004 \\
\end{array}$ \\
& 8 & 0.0232 & $0.0225 \pm 0.0002$ \\
& 10 & 0.0129 & $0.0124 \pm 0.0001$ \\
& & 0.00817 & $0.0079 \pm 0.0001$ \\
\hline Eq. (17) & 2 & 0.175 & $0.170 \pm 0.002$ \\
& 4 & 0.0364 & $0.0349 \pm 0.0003$ \\
& 6 & 0.0152 & $0.0149 \pm 0.0002$ \\
& 8 & 0.00832 & $0.0081 \pm 0.0001$ \\
& 10 & 0.00524 & $0.00510 \pm 0.00005$ \\
\hline \multirow{2}{*}{ Eq. (18) } & 2 & 0.134 & $0.126 \pm 0.001$ \\
& 4 & 0.0167 & $0.0162 \pm 0.0001$ \\
& 6 & 0.00497 & $0.00478 \pm 0.00005$ \\
& 8 & 0.00211 & $0.00204 \pm 0.00002$ \\
& 10 & 0.00108 & $0.00105 \pm 0.00001$ \\
& & & \\
\hline
\end{tabular}

vacuum above the emitting plane is, in the absence of the presence of the sphere, independent of position if the emittance from the plane is a constant according to Eq. (16). Since the sphere has a vanishingly small optical size, its presence does not affect this uniform photon density. Hence the collision density (scattering source) within the sphere is spatially uniform, and this implies that the flux of photons leaving the spherical surface is independent of position on the sphere. This is precisely the assumption made to incorporate a simple viewfactor treatment into the computation of the analytic bound. ${ }^{1}$ The same argument cannot be made when the emittance function, $F_{\mathrm{E}}(r)$, depends upon the spatial coordinate $r$. In this case, the viewfactor treatment used to obtain the analytic result represents an approximation. Thus the Monte Carlo (exact) results in Table 1 do not, nor should they, agree with the analytic estimate. The analytic result appears in general to be slightly lower than the Monte Carlo result, of the order of a few percent. However, as $h / R$ increases, the analytic estimate increases in accuracy.

Table 2 gives analogous results for $\sigma R=50$, and tests the accuracy of the optically thick sphere analytic bound. In this case, the use of simple viewfactors to produce the analytic result is, for all emittance functions, an approximation ' (the intensity leaving the sphere is not isotropic), and we should not expect this analytic result to agree with the Monte Carlo simulations in any case. We see that this is indeed confirmed in Table 2; the analytic estimate is consistently high compared to the Monte Carlo (exact) result, with the discrepancy again quite small, of the order of a few percent. Thus we conclude that the analytic results for the geometric factor, $G(0)$, reported in an earlier paper ${ }^{1}$ are quite accurate in both the optically thin and optically thick limits.

The next item we consider is the efficiency of the variance reduction techniques discussed in the last section. We performed a series of calculations using various combinations of these techniques to assess the computational time saving associated with each. All combinations employed the point detector estimator and are labeled $0-4$ according to: $0=$ none (except point detector estimator), 1 = source biasing, 2 = source biasing + Russian roulette, $3=$ source biasing + forced collision, 4 = source biasing + forced collision + Russian roulette. We found it absolutely necessary to employ the point detector estimator to achieve acceptable results in a reasonable amount of

Table 3. Efficiency of variance reduction for $\sigma R=0.01$ and $h / R=2$.

\begin{tabular}{|c|c|c|c|c|}
\hline $\begin{array}{c}\text { Bias } \\
\text { Option }\end{array}$ & $\mathbf{G ( 0 )}$ & $\begin{array}{c}\text { Percent } \\
\text { Error }\end{array}$ & $\begin{array}{c}\text { CPU } \\
\text { Time (s) }\end{array}$ & $\begin{array}{c}\text { Estimated } \\
\text { CPU Time (s) }\end{array}$ \\
\hline 0 & 0.113 & 5.7 & 89 & 2900 \\
1 & 0.113 & 2.8 & 96 & 750 \\
2 & 0.119 & 3.0 & 57 & 500 \\
3 & 0.116 & 1.8 & 8 & 25 \\
4 & 0.115 & 1.0 & 8 & 8 \\
\hline
\end{tabular}

Table 4. Efficiency of variance reduction for $\sigma R=0.01$ and $h / R=8$.

\begin{tabular}{|c|c|c|c|c|}
\hline $\begin{array}{c}\text { Bias } \\
\text { Option }\end{array}$ & G(U) & $\begin{array}{c}\text { Percent } \\
\text { Error }\end{array}$ & $\begin{array}{c}\text { CPU } \\
\text { Time (s) }\end{array}$ & $\begin{array}{c}\text { Estimated } \\
\text { CPU Time (s) }\end{array}$ \\
\hline 0 & 0.0072 & 7.1 & 750 & 38000 \\
1 & 0.0070 & 2.1 & 96 & 420 \\
2 & 0.0071 & 0.7 & 1250 & 600 \\
3 & 0.0070 & 1.0 & 12 & 12 \\
4 & 0.0070 & 1.0 & 11 & 11 \\
\hline
\end{tabular}


Table 5. Efficiency of variance reduction for $\sigma R=10$ and $h / R=2$.

\begin{tabular}{|c|c|c|c|c|}
\hline $\begin{array}{c}\text { Bias } \\
\text { Option }\end{array}$ & $\mathbf{G ( 0 )}$ & $\begin{array}{c}\text { Percent } \\
\text { Error }\end{array}$ & $\begin{array}{c}\text { CPU } \\
\text { Time (s) }\end{array}$ & $\begin{array}{c}\text { Estimated } \\
\text { CPU Time (s) }\end{array}$ \\
\hline 0 & 0.206 & 2.9 & 21 & 180 \\
1 & 0.197 & 3.1 & 62 & 600 \\
2 & 0.200 & 2.5 & 11 & 70 \\
4 & 0.208 & 2.5 & 11 & 70 \\
\hline
\end{tabular}

Table 6. Efficiency of variance reduction for $\sigma R=10$ and $h / R=8$.

\begin{tabular}{|c|c|c|c|c|}
\hline $\begin{array}{c}\text { Bias } \\
\text { Option }\end{array}$ & $\mathbf{G ( 0 )}$ & $\begin{array}{c}\text { Percent } \\
\text { Error }\end{array}$ & $\begin{array}{c}\text { CPU } \\
\text { Time (s) }\end{array}$ & $\begin{array}{c}\text { Estimated } \\
\text { CPU Time (s) }\end{array}$ \\
\hline 0 & 0.0112 & 2.2 & 309 & 1500 \\
1 & 0.0114 & 1.9 & 64 & 230 \\
2 & 0.0114 & 1.4 & 19 & 40 \\
\hline
\end{tabular}

computational time. We did perform a few calculations in a completely analog fashion, without employing the point detector estimator or any other variance reduction technique, to estimate the total importance of variance reduction, in particular that achieved using the point detector estimator. We discuss these results shortly. All of our calculations to test variance reduction strategies were performed using a constant plane emittance function given by Eq. (16). Further, all employed $\tau_{\text {eff }}$ as given by Eq. (34) to compute $G(0)$ according to Eq. (2). Our results are summarized in Tables 3-6. The column labeled "Percent Error" is the error corresponding to 1 $\mathrm{SD}$, and the CPU time, in seconds, is for execution on a Cray XMP. The absolute times are not particularly important; the relative times associated with each biasing option are of most interest. The column labeled "Estimated CPU Time" is the estimated execution time required to obtain a standard deviation corresponding to a $1 \%$ fractional error. This was estimated from actual computational times by assuming that the CPU time is inversely proportional to the square of the percentage error. It is this estimated CPU time which gives the estimated time saving factors associated with each biasing option.

Table 3 gives results for $\sigma R=0.01$ and $h / R=2$. This can be characterized as an optically thin sphere close to the emitting plane. By comparing the estimated CPU times, we can conclude that source biasing alone (option 1) yields an improvement of a factor of 4 , and that the subsequent use of Russian roulette (option 2) has a small effect beyond that. We also see that the forced collision technique coupled with source biasing (option 3) gives a factor of 30 improvement over source biasing alone (option 1), and that the subsequent use of Russian roulette (option 4) yields another factor of 3 . The overall computational savings from using all variance reduction techniques (option 4) over using none except the point detector estimator (option 0 ) is close to a factor of 400 .

Table 4 gives results for $\sigma R=0.01$ and $h / R=8$. This can be characterized as an optically thin sphere far from the emitting plane. We see that source biasing (option 1) yields a factor of 90 improvement, and Russian roulette (option 2) had little effect. This small effect, however, actually was in the direction of worse performance. Forced collisions (option 3) yielded another factor of 50 improvement, and again Russian roulette (option 4) had little effect. The overall computational savings from using all variance reduction techniques (option 4) over using none except the point detector estimator (option 0 ) is approximately a factor of 3500 . For this problem we considered a complete analog simulation, not using the point detector estimator. Rather we used a simple tally surface as described in the last section. This problem could not be run anywhere near the length of time required to obtain $1 \%$ statistical error because of the inefficiency of using a small tally surface. From our partial results, we estimated that $4 \times 10^{14}$ histories would be required to achieve a $1 \%$ SD. This translates into about 3 yr of Cray computational time, as contrasted with $11 \mathrm{sec}$ using the point detector estimator and our other variance reduction techniques. Comparing this estimated $3 \mathrm{yr}$ with the $38,000 \mathrm{sec}$ in Table 4 , we see the absolute necessity of using the point detector estimator for our problem; it alone gives an estimated computational savings for this problem of a factor of 2500 .

Table 5 gives results for $\sigma R=10$ and $h / R=2$. This can be characterized as an optically thick sphere close to the emitting plane. We see that source biasing without Russian roulette (option 1) degrades performance, because of the fact that the sphere is close to the emitting plane and large fluctuations are caused by preferentially following photons generated far from the sphere. This degradiation is not seen for thin spheres close to the emitting plane (see Table 3), probably because of the fact that the location of a first collision is not as important for thin spheres as for thick spheres. We also see that the use of Russian roulette in conjunction with source biasing (option 
2) yields an improvement factor of nearly 3 over using no variance reduction other than the point detector estimator (option 0 ). For a thick sphere, the use of forced collisions should have virtually no affect since nearly all of the photons hitting the sphere suffer a collision with no forcing. This is born out in Table 5 by comparing the entries corresponding to options 2 and 4 .

Table 6 gives results for $\sigma R=10$ and $h / R=8$. This can be characterized as an optically thick sphere far from the emitting plane. For this problem we did not use the forced collision technique (options 3 and 4) since it would be expected to have no effect, and this was demonstrated in the prior problem (see Table 5). We see that source biasing alone (option 1) yields a factor of 6 improvement, and the subsequent use of Russian roulette (option 2) contributes another factor of 7. Source biasing is more effective here than in the problem summarized in Table 5 because of the decreased solid angle subtended by the sphere.

Based upon the results summarized here and other simulations we have run, the range of observed performance gains are roughly given as follows: source biasing, 1-10; Russian roulette, 1-10; forced collisions, 1-100; point-detector estimator, 1-2500. In general, the use of the point-detector estimator is the most effective variance-reduction technique. The exception to this statement is when $h / R \simeq 1$; i.e., when the sphere is nearly touching the emitting plane. In this case, the well known large variance for this estimator due to collisions close to the detector comes into play.

The next item we consider is the comparison of $\tau_{\mathrm{D}}$ and $\tau_{\mathrm{av}}$ as given by Eqs. (3) and (34) with $\tau_{\text {eff }}$ as defined by Eq. (35). It is the use of $\tau_{\text {eff }}$ for $\tau$ in Eq. (2) that gives the exact treatment of the first collision probability. On the other hand, one would hope to be able to use, with acceptable accuracy, $\tau_{\mathrm{D}}$ for $\tau$ in Eq. (2) since $\tau_{\mathrm{eff}}$ can only be found from a Monte Carlo simulation or a very complex analytic formulation involving multiple numerical integrations. ${ }^{1}$ The numerical results we give here are for a constant emittance function given by $\mathrm{Eq}$. (16). The results for the other two emittance functions given by Eqs. (17) and (18) are very much the same. Table 7 gives typical results, with the numbers in parentheses indicating the corresponding values for the first collision probability $1-\exp (-\tau)$. The statistical errors in the values of $\tau_{\mathrm{av}}$ and $\tau_{\text {eff }}$ reported in Table 7 are of the order of $1 \%$. We see that for optically thin spheres $(\sigma R=0.1)$ all three values of $\tau$ are essentially identical. For optically thick spheres $(\sigma R=10), \tau_{\mathrm{D}}$ and $\tau_{\mathrm{av}}$ agree very well, but both are substantially different from $\tau_{\text {eff }}$, as is to be expected. However, in this case the quantity of interest, $1-\exp (-\tau)$, is essentially unity since $\tau$ is large according to all three prescriptions. The first flight collision probability shows the greatest variation for spheres of intermediate optical thickness $(\sigma R=1)$. In this case we see that the use of $\tau_{\mathrm{D}}$ to compute $1-\exp (-\tau)$ yields a value 4 parts out of 70 greater than the true value computed with $\tau_{\text {eff }}$. Based upon this table and other results, we conclude that the use of the Dirac result, $\tau_{\mathrm{D}}$, to compute the first collision probability is very accurate in both limits of optically small and large spheres, and overestimates the true values by a maximum of no more than $10 \%$ for spheres of intermediate optical size.

The final item we consider is that which motivated this paper. We wished to ascertain, via exact (except for statistical errors) Monte Carlo simulations which of the two previously reported bounds

Table 7. The accuracy of using $\tau_{D}$ to compute the first collision probability.

\begin{tabular}{|c|c|c|c|c|}
\hline$\sigma \mathbf{R}$ & $\mathbf{h} / \mathbf{R}$ & $\tau_{\mathbf{D}}$ & $\tau_{\mathbf{2 v}}$ & $\tau_{\mathrm{cff}}$ \\
\hline 0.1 & 2 & $\begin{array}{c}0.133 \\
(0.125)\end{array}$ & $\begin{array}{c}0.134 \\
(0.125)\end{array}$ & $\begin{array}{c}0.132 \\
(0.124)\end{array}$ \\
\hline 1.0 & 2 & $\begin{array}{c}1.33 \\
(0.74)\end{array}$ & $\begin{array}{l}1.33 \\
(0.74)\end{array}$ & $\begin{array}{c}1.21 \\
(0.70)\end{array}$ \\
\hline 10.0 & 2 & $\begin{array}{l}13.3 \\
(1.00)\end{array}$ & $\begin{array}{l}13.3 \\
(1.00)\end{array}$ & $\begin{array}{c}5.24 \\
(0.99)\end{array}$ \\
\hline 0.1 & 6 & $\begin{array}{c}0.133 \\
(0.125)\end{array}$ & $\begin{array}{c}0.133 \\
(0.125)\end{array}$ & $\begin{array}{c}0.132 \\
(0.124)\end{array}$ \\
\hline 1.0 & 6 & $\begin{array}{c}1.33 \\
(0.74)\end{array}$ & $\begin{array}{l}1.34 \\
(0.74)\end{array}$ & $\begin{array}{c}1.22 \\
(0.70)\end{array}$ \\
\hline 10.0 & 6 & $\begin{array}{l}13.3 \\
(1.00)\end{array}$ & $\begin{array}{l}13.3 \\
(1.00)\end{array}$ & $\begin{array}{c}5.28 \\
(0.99)\end{array}$ \\
\hline
\end{tabular}


Table 8. The accuracy of an interpolation model to compute $G(0)$.

\begin{tabular}{|c|c|c|c|c|}
\hline $\begin{array}{c}\text { Emittance } \\
\text { Function } \\
\mathbf{F}_{\mathbf{E}^{(\mathbf{r})}}\end{array}$ & $\sigma \mathbf{R}$ & $\mathbf{h} / \mathbf{R}$ & $\begin{array}{c}\mathbf{G}(0) \\
\text { Monte Carlo } \\
\text { Using }{ }^{{ }^{2} \mathrm{D}}\end{array}$ & $\underset{\text { Interpolated }}{\mathbf{G}(\mathbf{0})}$ \\
\hline Eq. (16) & $\begin{array}{r}0.1 \\
1.0 \\
10.0 \\
0.1 \\
1.0 \\
10.0\end{array}$ & $\begin{array}{l}2 \\
2 \\
2 \\
6 \\
6 \\
6\end{array}$ & $\begin{array}{l}0.114 \\
0.127 \\
0.201 \\
0.0124 \\
0.0132 \\
0.0203\end{array}$ & $\begin{array}{l}0.113 \\
0.121 \\
0.191 \\
0.0125 \\
0.0132 \\
0.0196\end{array}$ \\
\hline Eq. (17) & $\begin{array}{r}0.1 \\
1.0 \\
10.0 \\
0.1 \\
1.0 \\
10.0\end{array}$ & $\begin{array}{l}2 \\
2 \\
2 \\
6 \\
6 \\
6\end{array}$ & $\begin{array}{l}0.0656 \\
0.0760 \\
0.143 \\
0.00691 \\
0.00744 \\
0.0128\end{array}$ & $\begin{array}{l}0.0619 \\
0.0698 \\
0.137 \\
0.00688 \\
0.00746 \\
0.0124\end{array}$ \\
\hline Eq. (18) & $\begin{array}{r}0.1 \\
1.0 \\
10.0 \\
0.1 \\
1.0 \\
10.0\end{array}$ & $\begin{array}{l}2 \\
2 \\
2 \\
6 \\
6 \\
6\end{array}$ & $\begin{array}{l}0.0450 \\
0.0537 \\
0.106 \\
0.00198 \\
0.00218 \\
0.00409\end{array}$ & $\begin{array}{l}0.0414 \\
0.0479 \\
0.103 \\
0.00197 \\
0.00205 \\
0.00395\end{array}$ \\
\hline
\end{tabular}

(thin and thick spheres) for $G(0)$ is closest to the true value for a given optical size, $\sigma R$, of the sphere. Typical Monte Carlo results for $G(0)$ are given in Table 8 for two values of $h / R$, namely 2 and 6 , and for three values of $\sigma R$, namely 0.1 (thin), 1.0 (intermediate), and 10.0 (thick). The statistical errors in these results are of the order of $1 \%$ (varying from 0.5 to $1.5 \%$ for different cases). A comparison of these results with the bounds given in Tables 1 and 2 shows that the optical size of the sphere must be quite large for $G(0)$ to approach the thick sphere bound. That is, except for $\sigma R$ very large, the true value of $G(0)$ is closer to the thin limit than the thick limit.

It would be very useful to have an interpolation formula for $G(0)$ of the form

$$
G(0)=\alpha G_{N}(0)+(1-\alpha) G_{K}(0),
$$

where $G_{N}(0)$ denotes the thin limit and $G_{K}(0)$ denotes the thick limit. The parameter $\alpha$ here is an interpolation parameter which is a function of $\sigma R$ and approaches unity as $\sigma R$ approaches zero, and approaches zero as $\sigma R$ increases without bound. In the Appendix, a simple model gives the result

$$
\alpha=\frac{2\left\{\left[\frac{1}{3}-E_{4}(T)\right]+\lambda\left[\frac{1}{2}-E_{3}(T)\right]-T E_{3}(T)\right\}}{(2 \lambda+T)\left[\frac{1}{2}-E_{3}(T)\right]},
$$

where $T=2 \sigma R / 3, \lambda=0.7104$ (the Milne problem extrapolation distance ${ }^{2}$ ), and $E_{n}(z)$ is the $n$th order exponential integral. Typical values of $\alpha$ according to Eq. (37) are $\alpha=0.998(\sigma R=0.1)$, $\alpha=0.930(\sigma R=1.0)$, and $\alpha=0.340(\sigma R=10.0)$. We see from these values that the thin limit is emphasized in Eq. (36) except for very large spheres.

The results of using Eqs. (36) and (37) to estimate $G(0)$ are also given in Table 8. We see that this model is reasonably, but not spectacularly, successful, generally underestimating the true value of $G(0)$. If the accuracy predicted by Eqs. (36) and (37) is acceptable, one then has a very simple way of estimating the reflected flux directly under the sphere. One uses the thin and thick bonds previously reported,' together with Eqs. (36) and (37), to compute $G(0)$. This result is used in Eq. (2), with $\tau$ taken as the Dirac estimate as given by Eq. (3), to compute $\Gamma(0)$. We note that $\tau_{\mathrm{D}}$ was used to compare the Monte Carlo values of $G(0)$ in Table 8 , and hence the approximation of using $\tau_{\mathrm{D}}$ was factored into the rest of the interpolation formula given in Eqs. (36) and (37). Finally, $F_{\mathrm{R}}(0)$, the reflected flux directly under the sphere, follows from Eq. (1).

Acknowledgements - This work was partially supported by the Lawrence Livermore National Laboratory. In addition, the work of the second author (GCP) was partially supported by the National Science Foundation. 


\section{REFERENCES}

1. G. C. Pomraning, JQSRT 39, 261 (1988).

2. K. M. Case, F. de Hoffmann, and G. Placzek, "Introduction to the Theory of Neutron Diffusion," Vol. I, Los Alamos Scientific Laboratory, Los Alamos, NM (1953).

3. G. C. Pomraning, The Equations of Radiation Hydrodynamics, Pergamon Press, Oxford (1973).

\section{APPENDIX}

\section{An Interpolation Model}

To model the interpolation parameter given in Eq. (37), we consider a purely isotropically scattering, sourcefree sphere of radius $R$ and constant cross section $\sigma$ as in the main body of the text. We first transform this sphere into an "equivalent" slab of thickness $T_{\mathrm{e}}$. The equivalence is achieved by maintaining the same Dirac chord length for the slab as for the sphere. For a body of volume $V$ and surface area $S$, the Dirac chord length $l_{\mathrm{D}}$ is given by ${ }^{2}$

$$
l_{\mathrm{D}}=4 \mathrm{~V} / \mathrm{S}
$$

which yields

$$
l_{\mathrm{D}}(\text { sphere })=4 R / 3 ; \quad l_{\mathrm{D}}(\text { slab })=2 T_{\mathrm{e}},
$$

and hence equivalence is established by taking $T_{\mathrm{e}}=2 R / 3$. If we envisage an isotropic intensity incident upon the left-hand edge of the slab, taken as $x=0$, the first collision source in the slab will have a spatial dependence proportional to $E_{2}(\sigma x)$, where $x$ is the slab spatial coordinate and $E_{n}(z)$ is the $n$th order exponential integral. We approximate the solution of the equation of transfer with this source by using the diffusion approximation.

If we introduce $z=\sigma x$ as an optical depth coordinate, the diffusion equation is simply $y^{2,3}$

$$
-\frac{1}{3} \frac{\mathrm{d}^{2} \phi(z)}{\mathrm{d} z^{2}}=E_{2}(z), \quad 0 \leqslant z \leqslant T,
$$

where $T=\sigma T_{\mathrm{e}}$ is the optical thickness of the slab. The Milne boundary conditions corresponding to no intensity incident upon the slab surfaces are given by ${ }^{3}$

$$
\begin{aligned}
& {[\phi(z)-\lambda \mathrm{d} \phi(z) / \mathrm{d} z]_{z=0}=0,} \\
& {[\phi(z)+\lambda \mathrm{d} \phi(z) / \mathrm{d} z]_{z-\tau}=0,}
\end{aligned}
$$

where $\lambda=0.7104 \ldots$ is the Milne extrapolation distance. ${ }^{2}$ The dependent variable $\phi(z)$ in Eqs. (A3)-(A5) is proportional to the photon density arising from the first collision source. These equations are easily solved for $\phi(z)$, and one can subsequently compute the photon flux $F(z)$ from Fick's law given by

$$
F(z)=-\frac{1}{3} \frac{\mathrm{d} \phi(z)}{\mathrm{d} z} .
$$

In particular, one finds that the fluxes leaving the slab through each surface are given by

$$
\begin{gathered}
F_{\text {out }}(0)=(1 / 2)-\beta, \\
F_{\text {out }}(T)=\beta-E_{3}(T),
\end{gathered}
$$

where

$$
\beta=\left\{\left[(1 / 3)-E_{4}(T)\right]+\lambda\left[(1 / 2)+E_{3}(T)\right]\right\} /(2 \lambda+T) .
$$

We associate the outgoing flux at $z=T, F_{\text {out }}(T)$, and an equal amount at $z=0$ with the thin sphere (slab) limit. The remainder of the flux exiting the slab at $z=0$ is associated with the thick sphere (slab) limit. If we define $\alpha$ as the thin limit fraction, we then have

$$
\alpha=2 F_{\text {out }}(T) /\left[F_{\text {out }}(0)+F_{\text {out }}(T)\right],
$$


or, using Eqs. (A7) and (A8),

$$
\alpha=2\left[\beta-E_{3}(T)\right] /\left[(1 / 2)-E_{3}(T)\right] .
$$

Using Eq. (A9) for $\beta$ in Eq. (A11), we find the expression for $\alpha$ given by Eq. (37) in the main body of the text. It is easily verified that $\alpha$ has the proper limiting behaviors, namely

$$
\alpha \underset{T \rightarrow 0}{\longrightarrow} 1 ; \quad \alpha \underset{T \rightarrow \infty}{\longrightarrow} 0 .
$$

